\title{
Formation of deeply bound pionic atoms and pion properties in nuclei
}

\author{
Natsumi Ikeno* \\ Department of Physics, Nara Women's University, Nara 630-8506, Japan \\ E-mail: jan_ikeno@cc.nara-wu.ac.jp

\section{Junko Yamagata-Sekihara}

Institute of Particle and Nuclear Studies, High Energy Accelerator Research Organization

(KEK), Ibaraki 305-0801, Japan

E-mail: yamajunepost.kek.jp

\section{Hideko Nagahiro}

Department of Physics, Nara Women's University, Nara 630-8506, Japan

E-mail: nagahiro@cc.nara-wu.ac.jp

\section{Satoru Hirenzaki}

Department of Physics, Nara Women's University, Nara 630-8506, Japan

E-mail: zaki@cc.nara-wu.ac.jp

We study theoretically the formation of the deeply bound pionic atoms in various cases to deduce the pion properties in nuclei precisely from the observables. We show the angular dependence of the pionic atom formation spectra of the ${ }^{122} \mathrm{Sn}\left(d,{ }^{3} \mathrm{He}\right)$ reactions in the forward angles. At each scattering angle, a different combination of the pion-bound and neutron-hole states dominates the spectrum because of the kinematical condition. We conclude that we can obtain information on the deeply bound pionic $2 p$ state in addition to the $1 s$ and $2 s$ states by observing the spectra in finite angles. We also consider the neutron-odd nucleus as a target nucleus of the formation reaction, because it has an advantage that the pionic atom is formed in the even-even nucleus, of which the ground state has no neutron-hole states. In the ${ }^{117} \mathrm{Sn}\left(d,{ }^{3} \mathrm{He}\right)$ reaction, we find that we can see clearly the peak structure of the pionic $1 s$ state formation with the ground state of the even-even nucleus ${ }^{116} \mathrm{Sn}$. The pionic atom in the even-even nucleus will not have additional shifts induced by the effects of the residual interaction between pion-bound and neutron-hole states. The observation of these pionic states is preferable for extracting the accurate information on the pion properties from data.

XV International Conference on Hadron Spectroscopy-Hadron 2013

4-8 November 2013

Nara, Japan

\footnotetext{
* Speaker.
} 


\section{Introduction}

Deeply bound pionic atom is a very interesting system to deduce pion properties at finite density and to obtain precise information on partial restoration of chiral symmetry in nuclei [1]. The deeply bound pionic states have been experimentally produced in the $\left(d,{ }^{3} \mathrm{He}\right)$ reactions with the $\mathrm{Pb}$ and $\mathrm{Sn}$ targets by following theoretical predictions. In Ref. [2], the energy shifts and widths of the pionic $1 s$ state have been measured in three $\mathrm{Sn}$ isotopes. From these observations, the changes of the pion decay constant $f_{\pi}$ and the chiral order parameter $\langle\bar{q} q\rangle$ in nuclei were concluded.

However, to develop further studies of the pion properties and symmetry restoration in nuclei, we need more systematic information on the bound state spectrum. It helps us to determine the pion-nucleus interaction uniquely by reducing systematic errors coming from the uncertainties of the neutron distribution in the pionic atom and the absolute energy calibration. From the precise knowledge of the pion-nucleus interaction, one can deduce the in-medium modification of the fundamental quantities related to chiral symmetry, such as the quark condensate.

In this paper, we report the theoretical studies of the formation of the deeply bound pionic atoms in various cases. We study the formation of deeply bound pionic atoms by the $\left(d,{ }^{3} \mathrm{He}\right)$ reaction in finite angles. At forward angle, the contribution from the pionic $s$ state formation with the neutron $s$ hole state is selectively populated because of the kinematical selection rule in the recoilless kinematics. On the other hand, we can expect to have the manifestation of the formation of the different pionic states in finite angles because of the finite momentum transfer.

We also consider the neutron-odd nucleus as a target nucleus of the formation reaction in addition to the even-even nucleus. So far, all theoretical and experimental studies have been concentrated on the even-even nuclear target cases. For the even-neutron nuclear target cases, since the final pionic states are the pion-particle plus neutron-hole $\left[\pi \otimes n^{-1}\right]$ states, we will have to take into account the residual interaction effects [3, 4] to deduce the pion-nucleus interaction precisely from the high precision experimental data. On the other hand, for the odd-neutron nuclear target cases, we expect to observe the pionic states with the ground state of the even-even nucleus with $J^{P}=0^{+}$, which will not be affected by the additional shifts induced by the residual interaction effects. The observation of pionic states free from these effects is very important to obtain more accurate information on pion properties in the nuclei from data.

\section{Formulation}

We calculate the formation cross sections of the pionic atoms in the $\left(d,{ }^{3} \mathrm{He}\right)$ reaction using the effective number approach. The contributions of the bound state formation and the quasi-free pion production in the $\left(d,{ }^{3} \mathrm{He}\right)$ cross section are written as,

$$
\left(\frac{d^{2} \sigma}{d E_{\mathrm{He}} d \Omega_{\mathrm{He}}}\right)_{A}^{\mathrm{lab}}=\left(\frac{d \sigma}{d \Omega_{\mathrm{He}}}\right)_{\text {ele spins }}^{\text {lab }}\left[\frac{\Gamma}{2 \pi} \frac{1}{\Delta E^{2}+\Gamma^{2} / 4} K N_{\mathrm{eff}}+\frac{2\left|\vec{p}_{\pi}^{A}\right| E_{\pi}^{A}}{\pi} K N_{\mathrm{eff}}\right],
$$

in the laboratory frame. Here, $\left(\frac{d \sigma}{d \Omega_{\mathrm{He}}}\right)_{\text {ele }}^{\text {lab }}$ indicates the elementary cross section for the $d+n \rightarrow$ ${ }^{3} \mathrm{He}+\pi^{-}$reaction in laboratory frame, which is extracted from the experimental data [5]. The 
calculation of the cross section is done in the heavy limit of the target nucleus with introducing a kinematical correction factor $K$ defined by

$$
K=\left[\frac{\left|\vec{p}_{\mathrm{He}}^{A}\right|}{\left|\vec{p}_{\mathrm{He}}\right|} \frac{E_{n} E_{\pi}}{E_{n}^{A} E_{\pi}^{A}}\left(1+\frac{E_{\mathrm{He}}}{E_{\pi}} \frac{\left|\vec{p}_{\mathrm{He}}\right|-\left|\vec{p}_{d}\right| \cos \theta_{d \mathrm{He}}}{\left|\vec{p}_{\mathrm{He}}\right|}\right)\right]^{\mathrm{lab}},
$$

where the momenta and energies with index $A$ are evaluated in the kinematics of the finite nuclear target, and all the kinematical variables are evaluated in the laboratory frame. The correction factor $K$ is important to estimate the angular dependence of the cross section.

In order to predict the realistic spectrum shape of the $\left(d,{ }^{3} \mathrm{He}\right)$ reaction, we take into account the normalization factor $\left(F_{O}\right)$ of the neutron state in the target nucleus and the relative strength $\left(F_{R}\right)$ of the nuclear excited levels of the daughter nucleus, which are determined using the spectroscopic strength obtained from the experimental data of the one-neutron pick-up process. As for the symbol of the spin sum $\sum_{\text {spins }}$, we use the different practical expressions for the even-neutron and the oddneutron nuclear target cases. The different expressions of the effective number $N_{\text {eff }}$ are also used for the even- and odd-neutron nuclear target cases. The details of the spin sum symbol and the effective numbers are explained in Refs. 67,

\section{Numerical results}

We study the formation of the deeply bound pionic atoms in the ${ }^{117} \mathrm{Sn}\left(d,{ }^{3} \mathrm{He}\right)$ and ${ }^{122} \mathrm{Sn}\left(d,{ }^{3} \mathrm{He}\right)$ reactions at the forward angle $\theta_{d \mathrm{He}}^{\mathrm{lab}}=0^{\circ}$ and at a finite angle $\theta_{d \mathrm{He}}^{\mathrm{lab}}=2^{\circ}$.

In Fig. 1, we show the calculated spectra for the pionic state formation at $\theta_{d \mathrm{He}}^{\mathrm{lab}}=0^{\circ}$ in the ${ }^{117} \mathrm{Sn}\left(d,{ }^{3} \mathrm{He}\right)$ and ${ }^{122} \mathrm{Sn}\left(d,{ }^{3} \mathrm{He}\right)$ reactions with the deuteron energy $T_{d}=500 \mathrm{MeV}$. In addition to the total spectra, the contributions of the bound pionic state formation and the quasi-free $\pi^{-}$and $\pi^{0}$ production are also shown separately. The dominant subcomponents are also indicated in the figure with their quantum number. In the ${ }^{117} \mathrm{Sn}\left(d,{ }^{3} \mathrm{He}\right)$ spectra (left figure), we find that we can clearly see the peak structure of the pionic $1 s$ state formation with the ground state of the eveneven nucleus ${ }^{116} \mathrm{Sn}$ as indicated in the figure as $\left[(1 s)_{\pi} \otimes 0_{\text {ground }}^{+}\right]$. The subcomponents coupled to the ground state of the daughter nucleus ${ }^{116} \mathrm{Sn}$ will not have additional shifts induced by the residual interaction effects. In the ${ }^{122} \mathrm{Sn}\left(d,{ }^{3} \mathrm{He}\right)$ spectra (right figure), the $1 s$ and $2 s$ pionic states coupled with the same $\left(3 s_{1 / 2}\right)_{n}^{-1}$ neutron hole state can be seen as the clear peak structures.

In both the ${ }^{117} \mathrm{Sn}\left(d,{ }^{3} \mathrm{He}\right)$ and ${ }^{122} \mathrm{Sn}\left(d,{ }^{3} \mathrm{He}\right)$ spectra, the contribution from the pionic $1 s$ state formation with picking-up one neutron from the $s_{1 / 2}$ state is found to be large because of the matching condition with the recoilless kinematics. We find that the total spectrum of the bound pionic state formation in the ${ }^{117} \mathrm{Sn}\left(d,{ }^{3} \mathrm{He}\right)$ reaction spreads over a wider energy range than that in the ${ }^{122} \mathrm{Sn}\left(d,{ }^{3} \mathrm{He}\right)$ reaction. This is because the excited levels of ${ }^{116} \mathrm{Sn}$ tend to have larger excitation energies $\left(E_{x}\right)$ than those of ${ }^{121} \mathrm{Sn}$.

We show in Fig. 2 the calculated spectra for the pionic atom formation at $\theta_{d \mathrm{He}}^{\mathrm{lab}}=2^{\circ}$ in the ${ }^{117} \mathrm{Sn}\left(d,{ }^{3} \mathrm{He}\right)$ and ${ }^{122} \mathrm{Sn}\left(d,{ }^{3} \mathrm{He}\right)$ reactions. Due to the large momentum transfer, the pionic $2 p$ state contributions become relatively larger than those at $\theta_{d \mathrm{He}}^{\text {lab }}=0^{\circ}$ in the both spectra, and consequently the total spectra are more complicated in finite angles. Especially, for the ${ }^{117} \mathrm{Sn}$ target case, there are a lot of nuclear excited levels for one-neutron pick-up process, and thus there are much more 

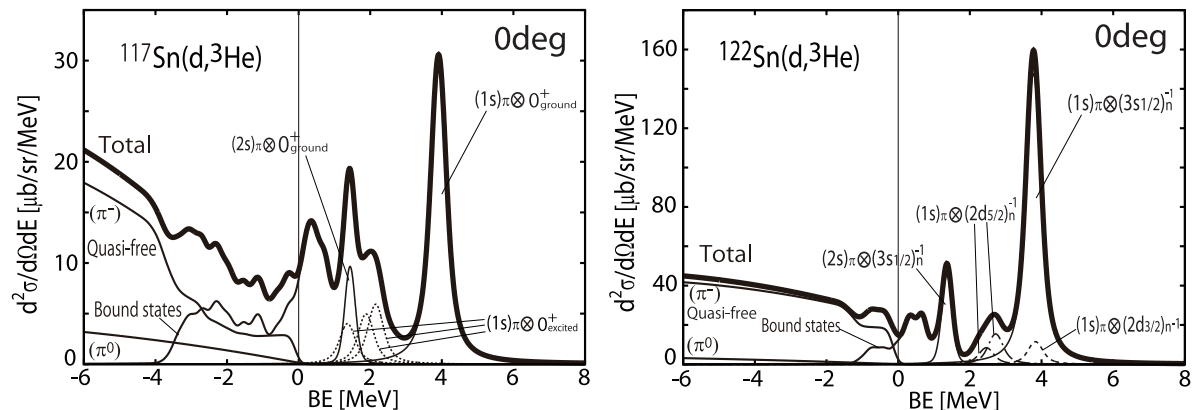

Figure 1: Calculated spectra for the formation of the pionic states at $\theta_{d \mathrm{He}}^{\text {lab }}=0^{\circ}$ in the ${ }^{117} \mathrm{Sn}\left(d,{ }^{3} \mathrm{He}\right)$ (left) and the ${ }^{122} \mathrm{Sn}\left(d,{ }^{3} \mathrm{He}\right)$ (right) reactions plotted as functions of the pion binding energy [7]. The dominant subcomponents are also shown in the figures with quantum numbers indicated as $\left[(n \ell)_{\pi} \otimes J^{P}\right]$ in the ${ }^{117} \mathrm{Sn}\left(d,{ }^{3} \mathrm{He}\right)$ reaction and $\left[(n \ell)_{\pi} \otimes\left(n \ell_{j}\right)_{n}^{-1}\right]$ in the ${ }^{122} \mathrm{Sn}\left(d,{ }^{3} \mathrm{He}\right)$ reaction, respectively. The instrumental energy resolution is assumed to be $300 \mathrm{keV}$ FWHM.
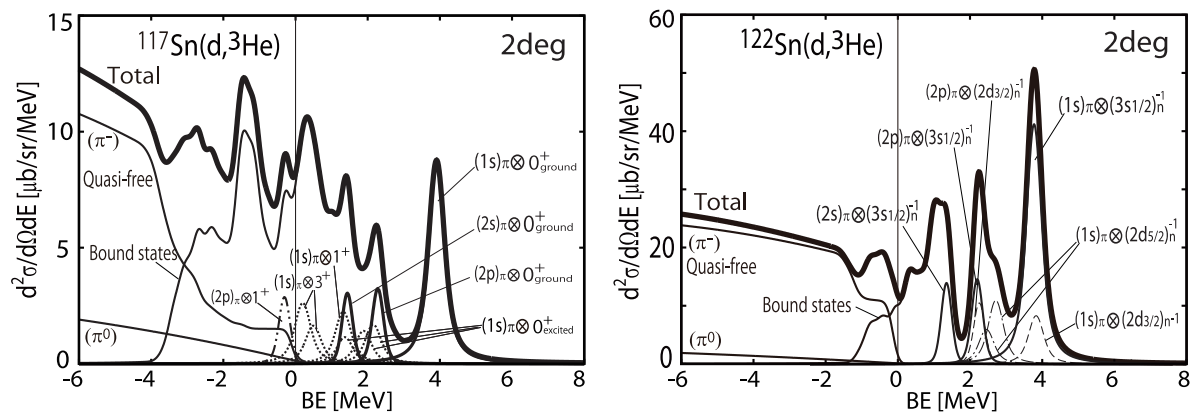

Figure 2: Calculated spectra for the formation of the pionic states at $\theta_{d \mathrm{He}}^{\text {lab }}=2^{\circ}$ in the ${ }^{117} \mathrm{Sn}\left(d,{ }^{3} \mathrm{He}\right)$ (left) and the ${ }^{122} \mathrm{Sn}\left(d,{ }^{3} \mathrm{He}\right)$ (right) reactions plotted as functions of the pion binding energy. In the ${ }^{117} \mathrm{Sn}\left(d,{ }^{3} \mathrm{He}\right)$ spectra, the contributions shown by thin lines and dots around B.E. $=-2 \sim 3 \mathrm{MeV}$ indicate a part of numerous subcomponents such as $\left[(1 s)_{\pi} \otimes 0_{\text {excited }}^{+}\right],\left[(1 s)_{\pi} \otimes 1^{+}\right],\left[(2 p)_{\pi} \otimes 1^{+}\right]$, and so on.

subcomponents which contribute to the $\left(d,{ }^{3} \mathrm{He}\right)$ spectra than those of ${ }^{122} \mathrm{Sn}$ target case. We show in Fig. 2 (left) some contributions from $\left[(1 s)_{\pi} \otimes 0_{\text {excited }}^{+}\right],\left[(1 s)_{\pi} \otimes 1^{+}\right],\left[(2 p)_{\pi} \otimes 1^{+}\right]$and so on as examples of numerous subcomponents of the ${ }^{117} \mathrm{Sn}\left(d,{ }^{3} \mathrm{He}\right)$ reaction. As naturally expected, the total spectrum of the ${ }^{117} \mathrm{Sn}\left(d,{ }^{3} \mathrm{He}\right)$ reaction is more complicated than that of the ${ }^{122} \mathrm{Sn}\left(d,{ }^{3} \mathrm{He}\right)$ reaction.

Nevertheless, we stress here that the spectra of the ${ }^{117} \mathrm{Sn}\left(d,{ }^{3} \mathrm{He}\right)$ reaction have the important advantage over that of the ${ }^{122} \mathrm{Sn}\left(d,{ }^{3} \mathrm{He}\right)$ reaction for getting the precise information on the pionic $1 s$ state. As we can see from Figs. 1 1 and 2, the largest peak produced by the contributions of the pionic $1 s$ state appears at B.E. $\simeq 3.9 \mathrm{MeV}$ in the both spectra of the ${ }^{117} \mathrm{Sn}$ and ${ }^{122} \mathrm{Sn}$ targets. For the ${ }^{122} \mathrm{Sn}$ target case, two subcomponents of $\left[(1 s)_{\pi} \otimes\left(3 s_{1 / 2}\right)_{n}^{-1}\right]$ and $\left[(1 s)_{\pi} \otimes\left(2 d_{3 / 2}\right)_{n}^{-1}\right]$ are included in the largest peak because of the small difference $(\sim 60 \mathrm{keV})$ between the separation energies of these two neutron levels in ${ }^{121} \mathrm{Sn}[8]$. The contribution of the $\left[(1 s)_{\pi} \otimes\left(2 d_{3 / 2}\right)_{n}^{-1}\right]$ subcomponent becomes relatively larger in finite angles because of the kinematical selection rule with the finite momentum transfer and it gives a non-negligible contribution. On the other hand, we find in Fig. 2 (left) that the $\left[(1 s)_{\pi} \otimes 0_{\text {ground }}^{+}\right]$subcomponent dominates the isolated peak structure at B.E. $\simeq 3.9 \mathrm{MeV}$ even at the finite angle $\theta_{d \mathrm{He}}^{\text {lab }}=2^{\circ}$ in the ${ }^{117} \mathrm{Sn}$ target case. This is because that the excited level has the large separation energy $(\sim 1.3 \mathrm{MeV})$ from the ground state in ${ }^{116} \mathrm{Sn}$. Therefore, we can say that the 
spectrum of the ${ }^{117} \mathrm{Sn}\left(d,{ }^{3} \mathrm{He}\right)$ reaction is more suited for the observation of the single subcomponent than that of the ${ }^{122} \mathrm{Sn}\left(d,{ }^{3} \mathrm{He}\right)$ reaction. In addition, this pionic $1 s$ state formation with the ground state of the even-even nucleus $\left[(1 s)_{\pi} \otimes 0_{\text {ground }}^{+}\right]$does not have any residual interaction effects.

We also find that the absolute values of the calculated cross sections in the ${ }^{117} \mathrm{Sn}\left(d,{ }^{3} \mathrm{He}\right)$ reaction are significantly smaller than those in the ${ }^{122} \mathrm{Sn}\left(d,{ }^{3} \mathrm{He}\right)$ reaction. This is because the values of the normalization factor $F_{O}$ and the relative strength $F_{R}$ of ${ }^{117} \mathrm{Sn}$ are smaller than those of the ${ }^{122} \mathrm{Sn}$ target case [7].

\section{Summary}

We have studied theoretically the formation of deeply bound pionic atoms and showed the ${ }^{117} \mathrm{Sn}\left(d,{ }^{3} \mathrm{He}\right)$ and ${ }^{122} \mathrm{Sn}\left(d,{ }^{3} \mathrm{He}\right)$ spectra at $\theta_{d \mathrm{He}}^{\mathrm{lab}}=0^{\circ}$ and $\theta_{d \mathrm{He}}^{\mathrm{lab}}=2^{\circ}$. We have found that the calculated spectra for the ${ }^{117} \mathrm{Sn}$ target clearly show the isolated peak structure with the signle subcomponent of the pionic $1 s$ state formation with the ground state of the even-even nucleus $\left(\left[(1 s)_{\pi} \otimes 0_{\text {ground }}^{+}\right]\right)$, which has no residual interaction effects. Thus, the formation of this pionic $1 s$ state is preferable to extract the most accurate information on the parameter of the QCD symmetry from the observation. The experiment for the pionic atom formation on the odd-neutron nuclear target will be performed at RIBF/RIKEN in near future [9].

We have also found that the ${ }^{122} \mathrm{Sn}\left(d,{ }^{3} \mathrm{He}\right)$ spectra are dominated by the subcomponents including $(2 p)_{\pi}$ state at larger angles $\theta_{d \mathrm{He}}^{\text {lab }} \geq 2^{\circ}$, while they are dominated by the $(1 s)_{\pi}$ and $(2 s)_{\pi}$ states in forward angles. Thus, we conclude that we can obtain information on the deeply bound pionic $2 p$ state in addition to the $1 s$ and $2 s$ states by observing the spectra in finite angles. As indicated in Ref. [8], the observation of several deeply pionic bound states in a certain nucleus will help to deduce precise information on the pion properties and the chiral dynamics at finite density. This sort of experiments has been performed at RIBF/RIKEN [10]. In near future, we will deduce more accurate pion properties in nuclei from data.

\section{References}

[1] T. Yamazaki, S. Hirenzaki, R.S. Hayano and H. Toki, Phys. Rep. 514 (2012) 1.

[2] K. Suzuki et al., Phys. Rev. Lett. 92 (2004) 072302.

[3] S. Hirenzaki, H. Kaneyasu, K. Kume, H. Toki and Y. Umemoto, Phys. Rev. C 60 (1999) 058202.

[4] N. Nose-Togawa, H. Nagahiro, S. Hirenzaki and K. Kume, Phys. Rev. C 71 (2005) 061601(R).

[5] M. Betigeri et al., Nucl. Phys. A 690 (2001) 473.

[6] N. Ikeno, H. Nagahiro and S. Hirenzaki, Eur. Phys. J. A 47 (2011) 161.

[7] N. Ikeno, J. Yamagata-Sekihara, H. Nagahiro and S. Hirenzaki, Prog. Theor. Exp. Phys. (2013) 063D01.

[8] N. Ikeno, R. Kimura, J. Yamagata-Sekihara, H. Nagahiro, D. Jido, K. Itahashi, L.S. Geng and S. Hirenzaki, Prog. Theor. Phys. 126 (2011) 483.

[9] K. Itahashi et al., Exp. proposal NP0702-RIBF-027 for RIBF, December (2006); Exp. proposal NP0802-RIBF-054 for RIBF, January (2008).

[10] T. Nishi, private communication. 